\title{
Growth outcomes of small for gestational age preterm infants before and after implementation of an exclusive human milk-based diet
}

\author{
Lindsay Fleig $\mathbb{D}^{1,2} \cdot$ Joseph Hagan ${ }^{1} \cdot$ Martin L. Lee $\mathbb{D}^{3,4} \cdot$ Steven A. Abrams $\mathbb{D}^{5} \cdot$ Keli M. Hawthorne ${ }^{5} \cdot$ Amy B. Hair $\mathbb{D}^{2}$
}

Received: 11 January 2021 / Revised: 30 March 2021 / Accepted: 28 April 2021 / Published online: 19 May 2021

(c) The Author(s) 2021. This article is published with open access

\begin{abstract}
Objective Small for gestational age (SGA) preterm infants (PT) are at greatest risk for growth failure. Our objective was to assess the impact of an exclusive human milk diet (HUM) on growth velocities and neonatal morbidities from birth to discharge in a SGA population.

Study design Multicenter, retrospective cohort study, subgroup analysis of SGA PT comparing a cow's milk diet (CMD) with HUM diet.

Results At birth 420 PT were classified as SGA (197 CMD group, 223 HUM group). Demographics and anthropometric measurements were similar. HUM group PT showed improvement in length $Z$ score at discharge $(p=0.024)$ and reduction in necrotizing enterocolitis (NEC) $(p=0.004)$.

Conclusion SGA PT fed a HUM diet had significantly decreased incidence of NEC, surgical NEC, and late-onset sepsis. Due to concerns about growth in a HUM diet, it is reassuring SGA infants fed the HUM diet had similar growth to CMD diet with trends toward improvement.
\end{abstract}

\section{Introduction}

Previously, our group published a multicenter retrospective cohort study comparing infants who received a cow's milk diet (CMD) of mother's own milk (MOM) fortified with cow's milk-based fortifier (CMF) and/or preterm formula to infants who received an exclusive human milk diet (HUM) of MOM and/or donor human milk (DHM) fortified with human milk-based fortifier (HMBF) [1, 2]. This pre-post

Lindsay Fleig

lindsay.n.fleig@uth.tmc.edu

1 Department of Pediatrics, Division of Neonatology, University of Texas at Houston Health Science Center, Children's Memorial Hermann Hospital, Houston, TX, USA

2 Department of Pediatrics, Section of Neonatology, Baylor College of Medicine, Texas Children's Hospital, Houston, TX, USA

3 Prolacta Bioscience, Duarte, CA, USA

4 Department of Biostatistics, UCLA Fielding School of Public Health, Los Angeles, CA, USA

5 Department of Pediatrics, Dell Medical School, University of Texas at Austin, Austin, TX, USA cohort study demonstrated improved outcomes in necrotizing enterocolitis (NEC), late-onset sepsis, and bronchopulmonary dysplasia (BPD) after implementation of the HUM diet. Infants born small for gestational age (SGA), defined as $<10$ th percentile for weight, are at higher risk for postnatal growth failure at discharge [3-6]. Our previous study included both SGA and appropriate for gestational age (AGA) infants in their cohorts.

The American Academy of Pediatrics, Committee on Nutrition updated their policy statement in December 2017 on breastfeeding and DHM use specific for high-risk preterm infant populations [7]. DHM use is now widely used in the US for preterm infants weighing less than $1500 \mathrm{~g}$ birth weight (BW) when MOM is not available or is contraindicated. MOM is always preferred because of the known biological components affected by pasteurization used in DHM. There are significant short- and long-term beneficial effects of feeding preterm infants a HUM diet compared to a mixed CMD diet, including decreased late-onset sepsis, reduction in BPD, mortality, and decreased risk of developing NEC supported by multiple published studies comprising of randomized controlled trials [8-11].

However, there still remain concerns about the growth of infants receiving a HUM diet, as human milk must be 
fortified for the most high-risk and premature infants to achieve adequate nutrient intake [12-14]. Hair et al. illustrated that the use of a HUM diet was safe and did not negatively impact growth of infants at a single study site in infants $<1250 \mathrm{~g} \mathrm{BW}$ [15]. Human milk should be fortified with protein, minerals, and vitamins to ensure optimal nutrient infant for infants weighing $<1500 \mathrm{~g} \mathrm{BW}$. It is established that both MOM and DHM have large variability in nutritional content in terms of calories per ounce, protein, carbohydrate, and fat content. Therefore, in order to avoid postnatal growth failure, a diet with human milk, either MOM or DHM, must be supplemented with fortification, either CMF or HMBF.

The primary aim of this study is to assess the impact of the HUM diet on anthropometric growth velocities from birth to discharge in the SGA infant population. The secondary outcomes assessed focus on the impact of the HUM diet on neonatal morbidities, specifically NEC and mortality, in this SGA infant population.

\section{Methods}

In a previously published multicenter, retrospective cohort study, infants who received a CMD diet of MOM fortified with CMF and/or preterm formula were compared to growth of infants who received a newly introduced HUM diet feeding protocol consisting of MOM and/or DHM with HMBF [1, 2]. Groups compared were infants fed a CMD diet to infants fed a HUM diet. All infants $<1250 \mathrm{~g} \mathrm{BW}$ and SGA were included. SGA was defined as $<10$ th percentile for weight in grams at birth [3]. Excluded infants had major congenital anomalies, died in the first $12 \mathrm{~h}$ of life or were transferred in from an outside hospital after 7 days of life. The Institutional Review Board at each study site approved this retrospective study.

\section{Study outcomes}

Study data were collected for approximately 2 years prior to and after introduction of a HUM diet. Primary data were extracted from the electronic medical record and included infant diet, demographics, growth parameters, and outcomes. Each study site had detailed documentation of feeding protocol and time frame for data collection [1]. Participating centers were Baylor College of Medicine (BCM) (Houston, TX), Good Samaritan San Jose Hospital (GSH) (San Jose, CA), Northwestern Prentice Women's Hospital (Chicago, IL), and Winnie Palmer Hospital (Orlando, FL). BCM was the largest trial contributor with $62 \%$ infants; however, all sites were level 4 NICUs in both academic and private institutions. There were differences among the sites with respect to specific feeding protocols documented in detail. Full feedings were considered 140-160 ml/kg/day [1]. At BCM in the HUM diet group, enteral feeds were started with trophic feeds of $20 \mathrm{ml} / \mathrm{kg} /$ day for 3 days, and advanced by $20 \mathrm{ml} / \mathrm{kg} /$ day as tolerated to goal of $140-160 \mathrm{ml} / \mathrm{kg} / \mathrm{day}$. HMBF was added (Prolacta Biosciences, Industry, CA) at $60 \mathrm{ml} / \mathrm{kg} /$ day volume for an additional $4 \mathrm{kcal} / \mathrm{oz}$, at $100 \mathrm{ml} / \mathrm{kg} / \mathrm{day}$ at for an additional $6 \mathrm{kcal} / \mathrm{oz}$, and if weight gain was $<15 \mathrm{~g} / \mathrm{kg} /$ day, an additional $8-10 \mathrm{kcal} / \mathrm{oz}$ was provided. At BCM in the CMD diet group, fortification did not start until feeds were at $100 \mathrm{ml} / \mathrm{kg} / \mathrm{day}$. At the GSH, the HUM group feeds were fortified at $100 \mathrm{ml} / \mathrm{kg} /$ day with $4 \mathrm{kcal} / \mathrm{oz}$, and then at $150 \mathrm{ml} / \mathrm{kg} /$ day an additional $6 \mathrm{kcal} / \mathrm{oz}$ was provided, and if growth failure, addition of $8-10 \mathrm{kcal} / \mathrm{oz}$ was provided. The CMD diet group at GSH followed the same protocol as BCM. At Northwestern and Winne Palmer Hospital, the HUM diet group was fortified with an additional $4 \mathrm{kcal} / \mathrm{oz}$ once feeds reached $100-120 \mathrm{ml} / \mathrm{kg} / \mathrm{day}$, and advanced to $6 \mathrm{kcal} / \mathrm{oz}$ if weight gain was $<15 \mathrm{~g} / \mathrm{kg} / \mathrm{day}$, and further advanced to $8 \mathrm{kcal} / \mathrm{oz}$. In the CMD diet group at Northwestern followed the same protocol as BCM. At Winne Palmer Hospital, the CMD diet group was fortified approximately at $120-150 \mathrm{ml} / \mathrm{kg} / \mathrm{day}$. All infants, regardless of whether SGA or AGA, received the same feeding protocols at each institution, and no specific feeding protocol was followed for SGA infants.

The original primary study outcomes have been previously published for the entire cohort consisting of both SGA and AGA infants [1]. In this subgroup analysis of the original data, we investigated the SGA cohort alone specific for growth and neonatal outcomes. Growth velocities from birth to discharge were calculated and compared between CMD diet and HUM diet groups. Weight velocity was calculated in in grams per kilogram per day (g/kg/day) using the exponential method [16]. Length velocity was calculated in centimeters per day (cm/day). Head circumference (HC) velocity was calculated in centimeters per day (cm/day). $Z$ scores were calculated for the growth velocities using either Fenton growth curves if infants were discharged home before 50 weeks' postmenstrual age (PMA) or World Health Organization growth curves for infants discharged home after 50 weeks' PMA [3, 17]. Growth data were adjusted for length of stay, antenatal steroids, and by study site.

\section{Outcomes defined}

PMA at discharge was defined as the number of weeks and days added to gestational age at birth. As described by the Fenton growth curve, SGA was defined as BW in grams < 10th percentile and AGA was defined as BW in grams between the 10th and 90th percentiles [3]. Common 
neonatal outcomes measured included NEC defined as Bell's stage IIA or greater with presence of pneumatosis intestinalis on abdominal radiograph reviewed by a pediatric radiologist [18]. Surgical NEC was defined as NEC requiring surgical intervention within the acute phase of illness [1]. All NEC cases were reviewed individually at time of data collection to delineate between spontaneous intestinal perforations versus NEC. BPD was defined as mild BPD in infants $<32$ weeks' gestation with need for supplemental oxygen for greater than or equal to 28 days, moderate BPD was defined as requiring supplemental oxygen for greater than or equal to 28 days with less than $30 \% \mathrm{FiO}_{2}$ at 36 weeks' PMA, and severe BPD was defined as requiring supplemental oxygen for greater than or equal to 28 days with greater than or equal to $30 \%$ $\mathrm{FiO}_{2}$ or positive pressure at 36 weeks' PMA [19]. Lateonset sepsis was defined as a positive blood culture obtained after $72 \mathrm{~h}$ of life. Patent ductus arteriosus (PDA) was measured by echocardiogram read by a boardcertified pediatric cardiologist. Severe intraventricular hemorrhage was diagnosed by head ultrasound read by a board-certified pediatric radiologist using the Papile grading method [20].

\section{Statistical analysis}

The distributions of quantitative variables were summarized using the median and interquartile range with diet group comparisons made using the Wilcoxon rank-sum test. Categorical variables were compared between the study groups using Fisher's exact test. A 5\% significance level was used for all comparisons.

\section{Results}

Of the original 1587 infants among the four study centers, there were 420 infants who were classified as less than the 10th percentile, defined with Fenton growth curves, included in this analysis [1,3]. There were 197 infants in the CMD group, pre-initiation of the HUM feeding protocol, and 223 infants in the HUM group, post initiation of the HUM feeding protocol. Infant demographics and anthropometric measurements were similar (Table 1).

The primary outcomes for growth measurements between the CMD and HUM groups are shown in Table 2. Length discharge $Z$ score was greater on the average in the HUM group $(p=0.024)$. Although not significant, all other parameters (velocity, discharge $Z$ score, and change in $Z$ score from birth to discharge) for weight, length, and $\mathrm{HC}$ were numerically superior in the HUM group. Diagnosis of SGA at discharge was not significantly different between the groups $(p=0.14)$. Of the original 197 infants
Table 1 Small for gestational age (SGA) infants' characteristics $(n=420)$.

\begin{tabular}{|c|c|c|c|}
\hline & CMD $(n=197)$ & HUM $(n=223)$ & $p$ value \\
\hline $\mathrm{Male}^{\mathrm{a}}$ & $104(53)$ & $116(52)$ & 0.922 \\
\hline \multicolumn{4}{|l|}{ Race $^{\mathrm{a}}$} \\
\hline Black & $72(37)$ & $76(34)$ & 0.610 \\
\hline Hispanic & $46(23)$ & $45(20)$ & 0.477 \\
\hline White & $63(32)$ & $86(39)$ & 0.184 \\
\hline Other & $16(8)$ & $16(7)$ & 0.717 \\
\hline Gestational age (weeks) ${ }^{\mathrm{b}}$ & $28.0(26.0,30.2)$ & $28.0(26.0,30.0)$ & 0.516 \\
\hline Birth weight $(\mathrm{g})^{\mathrm{b}}$ & $705.0(566.0,923.0)$ & $760.0(580.0,965.0)$ & 0.215 \\
\hline Birth weight $Z$ score $^{\mathrm{b}}$ & $-1.5(-1.8,-1.2)$ & $-1.4(-1.8,-1.1)$ & 0.778 \\
\hline Birth length $(\mathrm{cm})^{\mathrm{b}}$ & $32.0(30.0,35.0)$ & $33.0(30.5,36.0)$ & 0.193 \\
\hline Birth length $Z$ score $^{\mathrm{b}}$ & $-1.6(-2.3,-1.0)$ & $-1.6(-2.2,-1.0)$ & 0.594 \\
\hline $\begin{array}{l}\text { Birth head circumference } \\
(\mathrm{cm})^{\mathrm{b}}\end{array}$ & $23.5(21.5,26.0)$ & $24.0(21.5,26.0)$ & 0.497 \\
\hline $\begin{array}{l}\text { Birth head circumference } \\
Z \text { score }^{b}\end{array}$ & $-1.4(-2.1,-1.0)$ & $-1.5(-2.0,-0.9)$ & 0.712 \\
\hline Multiple gestation ${ }^{a}$ & $40(20)$ & $55(25)$ & 0.297 \\
\hline SGA at birth ${ }^{\mathrm{a}}$ & $197(100)$ & $223(100)$ & 1.000 \\
\hline Antenatal steroids ${ }^{\mathrm{a}}$ & $162(82)$ & $187(84)$ & 0.697 \\
\hline
\end{tabular}

${ }^{a}$ Frequency (\%), Fisher's exact test $p$ value.

${ }^{\mathrm{b}}$ Median (interquartile range), Wilcoxon rank-sum test $p$ value.

in the CMD group, 168 infants $(85 \%)$ remained SGA at discharge and of the original 223 infants in the HUM group, $201(90 \%)$ remained SGA at discharge. Length of stay was not significantly different between the groups $(p=0.91)$.

The neonatal morbidities for the CMD and HUM groups are presented in Table 3. Among the SGA infants in this study, late-onset sepsis $(p=0.017)$, NEC $(p=0.004)$, and surgical NEC (0.045) were significantly lower in the HUM group. In our SGA population, the number needed to treat is 11.1 infants to prevent one case of NEC and 20 infants to prevent one case of surgical NEC. Death was similar among the groups with 36 deaths (18.2\%) in the CMD group compared to 37 deaths $(16.6 \%)$ in the HUM group $(p=0.70)$. In addition, PDA $(p=0.078)$, retinopathy of prematurity at any stage $(p=$ $0.18)$, and BPD $(p=0.27)$ rates were lower in the HUM group, but not significantly so. The HUM group required a median of 2 fewer mechanical ventilation days $(p=0.12)$.

\section{Discussion}

Use of a HUM diet in SGA infants improved length discharge $Z$ score, as evidenced previously in other studies $[15,21]$. In addition, use of a HUM diet in SGA infants showed decreased late-onset sepsis, medical and surgical NEC cases, thus providing further validation for the HUM diet to reduce NEC $[8,22]$. 
Table 2 Small for gestational age (SGA) infants' primary outcomes for growth parameters $(n=420)$.

\begin{tabular}{|c|c|c|c|}
\hline & $\mathrm{CMD}(n=197)$ & HUM $(n=223)$ & $p$ value \\
\hline Discharge weight $(\mathrm{g})^{\mathrm{a}}$ & $\begin{array}{l}2385.0 \\
(1920.0,3255.0)\end{array}$ & $\begin{array}{l}2390.0 \\
(1980.0,3045.0)\end{array}$ & 0.598 \\
\hline Discharge weight $Z$ score $^{\mathrm{a}}$ & $-2.4(-3.2,-1.6)$ & $-2.3(-3.1,-1.8)$ & 0.829 \\
\hline Weight $\Delta Z$ score $^{\mathrm{a}}$ & $-0.9(-1.5,-0.1)$ & $-0.8(-1.3,-0.4)$ & 0.915 \\
\hline Weight velocity $(\mathrm{g} / \mathrm{d})^{\mathrm{a}}$ & $19.6(15.8,23.2)$ & $19.7(16.5,22.6)$ & 0.843 \\
\hline Discharge length $(\mathrm{cm})^{\mathrm{a}}$ & $44.5(42.0,47.5)$ & $45.0(42.5,47.5)$ & 0.263 \\
\hline Discharge length $Z$ score ${ }^{\mathrm{a}}$ & $-3.3(-4.4,-2.2)$ & $-3.0(-3.9,-2.0)$ & 0.024 \\
\hline Length $\Delta Z$ score $^{\mathrm{a}}$ & $-1.4(-2.7,-0.4)$ & $-1.2(-2.1,-0.4)$ & 0.174 \\
\hline Length velocity $(\mathrm{cm} / \text { week })^{\mathrm{a}}$ & $0.9(0.7,1.0)$ & $0.9(0.8,1.1)$ & 0.210 \\
\hline Discharge head circumference $(\mathrm{cm})^{\mathrm{a}}$ & $32.5(30.6,35.3)$ & $33.0(31.0,35.0)$ & 0.828 \\
\hline Discharge head circumference $Z$ score $^{\mathrm{a}}$ & $-1.6(-2.4,-0.8)$ & $-1.5(-2.6,-0.7)$ & 0.563 \\
\hline Head circumference $\Delta Z$ score $^{\mathrm{a}}$ & $0.0(-0.9,0.8)$ & $-0.1(-1.0,0.4)$ & 0.286 \\
\hline Head circumference velocity $(\mathrm{cm} / \text { week })^{\mathrm{a}}$ & $0.7(0.6,0.8)$ & $0.7(0.6,0.8)$ & 0.179 \\
\hline SGA at birth ${ }^{\mathrm{a}}$ & $197(100)$ & $223(100)$ & 1.000 \\
\hline SGA at discharge ${ }^{a}$ & $168(85)$ & $201(90)$ & 0.137 \\
\hline Postmenstrual age at discharge (weeks) ${ }^{\mathrm{a}}$ & $39.6(37.2,43.8)$ & $39.9(37.4,43.4)$ & 0.901 \\
\hline Length of stay (days) $)^{\mathrm{a}}$ & $77.0(48.0,121.0)$ & $77.0(48.0,121.0)$ & 0.913 \\
\hline
\end{tabular}

${ }^{a}$ Median (interquartile range), Wilcoxon rank-sum test $p$ value.
Table 3 Small for gestational age (SGA) infants' secondary outcomes for neonatal morbidities $(n=420)$.

\begin{tabular}{lccc}
\hline & CMD $(n=197)$ & HUM $(n=223)$ & $p$ value \\
\hline NEC $^{\mathrm{a}}$ & $33(17)$ & $17(8)$ & 0.004 \\
Surgical NEC $^{\mathrm{a}}$ & $18(9)$ & $9(4)$ & 0.045 \\
PDA $^{\mathrm{a}}$ & $105(53)$ & $99(44)$ & 0.078 \\
ROP, any stage $^{\mathrm{a}}$ & $13(7)$ & $8(4)$ & 0.182 \\
Severe IVH $^{\mathrm{a}}$ & $15(8)$ & $15(7)$ & 0.850 \\
Death $^{\mathrm{a}}$ & $36(18.2)$ & $37(16.6)$ & 0.699 \\
BPD $^{\mathrm{a}}$ & $81(41)$ & $79(35)$ & 0.268 \\
Mechanical vent days $^{\mathrm{b}}$ & $9.0(2.0,43.0)$ & $7.0(0.0,41.0)$ & 0.121 \\
Late-onset sepsis $^{\mathrm{a}}$ & $53(27)$ & $38(17)$ & 0.017 \\
\hline
\end{tabular}

${ }^{a}$ Frequency (\%), Fisher's exact test $p$ value.

${ }^{\mathrm{b}}$ Median (interquartile range), Wilcoxon rank-sum test $p$ value.

This study is unique in that it is the first analysis in the SGA infant population comparing growth and neonatal morbidities at multiple institutions after implementation of a feeding protocol using the HUM diet. This study provides insight into differences among SGA infants and may provide evidence to target this high-risk population for a HUM diet. Multiple institutions and the relatively large number of infants enrolled in each group allows for increased generalizability of our results.

\section{Growth outcomes in SGA infants}

Further investigation into how the HUM diet impacts our most fragile and small infants, the SGA population, requires sincere equipoise. Controversy in evidence exists with respect to the HUM diet and growth failure. In a smaller retrospective study in a single level III NICU, infants $<1000 \mathrm{~g}$ BW fed the HUM diet had improved feeding intolerance but persistent growth failure despite adjustment for SGA [23]. SGA infants in this study were not evaluated separately from all extremely low birth weight (ELBW) infants. In addition, in Eibensteiner et al., 192 ELBW infants were evaluated at multiple centers and found no improvement in growth or neonatal morbidities, again without distinction of the SGA population [24]. SGA infants are at a higher risk for growth failure, longterm metabolic outcomes, higher risk for NEC and feeding intolerance, and therefore, should be evaluated separately. In contrast to these findings with specific focus on the SGA population by Hair et al., premature SGA infants fed the HUM diet exhibited greater catch-up growth without negative metabolic outcomes as compared to AGA infants [25]. In addition, premature SGA infants fed a HUM diet show lower insulin levels, no difference in adiposity in the groups, and improvement in body composition at 2-year follow-up [25]. Sullivan et al. showed that there was no difference in growth between infants fed HUM and infants fed a mixed CMD diet [8]. The ultimate goal in premature neonatal nutrition is to optimize enteral nutrition without increasing the risk of neonatal morbidities and mortality, such as life-threatening NEC. Inhospital growth is associated with long-term neurodevelopmental outcomes and improving growth may reduce overall morbidity associated with prematurity [26]. A systematic review illustrated improvement of 
neurodevelopmental outcomes with visual and cognitive benefits into adolescence with the increasing dose received of MOM in preterm infants [27].

The use of a HUM diet in infants $<1250 \mathrm{~g}$ BW (both SGA and AGA) is associated with a lower rate of NEC and decreased parenteral nutrition days $[1,8,9,11]$. However, there remains a paucity of evidence in the literature regarding a direct comparison of MOM fortified with CMF to the HUM in the most high-risk and fragile SGA premature infant population.

Postnatal growth failure is a common complication of prematurity and SGA infants are at even higher risk. There are concerns that infants classified as SGA at birth will have a high incidence of postnatal growth failure [26]. In a cohort of 1776 SGA infants, $97 \%$ of them remained SGA at 36 weeks' corrected age [5]. Similarly in our cohort, growth failure persisted at discharge in both groups, with a slightly improved relative reduction in SGA in the CMD group compared to HUM group, although the difference did not achieve statistical significance. Despite this smaller reduction in SGA at discharge, both groups showed similar growth. The SGA infants fed the HUM diet showed possible trends toward improvement, with only the length discharge $Z$ score being statistically significant. These clinically improved growth outcomes, although not statistically significant, in addition to decreased mortality support the use of DHM in the high-risk infant when MOM is not available and further supports the use of early and rapid advancement of fortification with HMBF for growth [8, 25].

In a study by Dusick et al., infants born with in utero growth failure less than $1000 \mathrm{~g}$ BW had a higher incidence of growth failure postnatally when compared to AGA [4]. Similarly, in a large retrospective study of 24,371 infants by Clark et al., growth restriction at discharge is inversely related to younger gestational age and weight at birth [6]. However, another study reports similar growth velocities when comparing SGA to AGA infants, perhaps because of factors other than fortification and attainment of full feedings [15]. These concerns for the SGA infant population make them of unique concern for further specialized investigation.

\section{Neonatal morbidities in SGA infants}

The reduction of NEC with use of a HUM diet evidenced in larger infants remains consistent in our cohort of SGA infants $[1,8,9,11]$. In this cohort there were $53 \%$ fewer cases of NEC in the HUM group. It is suspected that the effect of cow's milk in the premature infant diet is dose dependent with fewer days to full feedings and fewer ventilator days as the percentage of CMD decreased. Therefore, limiting exposure to CMF in the SGA population should be considered [22]. Moreover, significantly decreased incidence of late-onset sepsis was evident in the HUM group, consistent with previous reports [1].

\section{Limitations}

This study is limited by it being a secondary analysis of previously collected data gathered retrospectively and potentially unidentified time-dependent care changes. In addition, a retrospective study may have misclassification of NEC diagnosis, although the diagnosis was made by two separate physicians in the original study's Bell's Staging Criteria [18]. Despite these limitations, a significant difference in length discharge $Z$ score was evident, and other parameters favored the HUM group.

\section{Conclusion}

In this secondary analysis of a retrospective cohort study of SGA infants after the initiation of a HUM feeding protocol, those who received a HUM diet had significantly decreased incidence of NEC, surgical NEC, and late-onset sepsis. Although SGA infants fed a HUM diet had similar growth to CMD infants for most outcomes, length was improved in the HUM group. Due to concerns about growth in infants who receive a HUM diet, it is reassuring that SGA infants had similar growth to CMD with some possible trends toward improvement. Further investigation into the specialized needs of SGA infants to overcome postnatal growth failure is needed.

Acknowledgements The authors would like to acknowledge Pam Gordon, RN, at Texas Children's Hospital for her work with patient care and anthropometric measurements.

Author contributions Conceptualization: LF, MLL, SAA, ABH; methodology: LF, KMH, MLL, SAA, ABH; formal analysis: LF, JH, $\mathrm{KMH}, \mathrm{MLL}, \mathrm{SAA}, \mathrm{ABH}$; investigation: $\mathrm{LF}, \mathrm{ABH}$; data curation: $\mathrm{LF}$; writing — original draft preparation: LF; writing — review and editing: LF, KMH, MLL, SAA, ABH; visualization: $L F, A B H$; supervision: $\mathrm{LF}, \mathrm{ABH}$; project administration: $\mathrm{LF}, \mathrm{ABH}$; funding acquisition: $\mathrm{ABH}$.

\section{Compliance with ethical standards}

Conflict of interest $\mathrm{ABH}$ receives research support from Prolacta Bioscience ${ }^{\circledast}$ for the Human Milk Cream Length of Stay Study and the Human Milk Cardiac Study and research support from Fresenius Kabi for the SMOF Premature Infant Randomized Controlled Trial. She received no outside funding for this study. Participated in all study aspects, assisted in study design, data and statistical analysis, and cowrote the manuscript. MLL is an employee of Prolacta Bioscience. $\mathrm{KMH}$ is a member of Prolacta's speaker bureau. All other authors declare no conflict of interest.

Publisher's note Springer Nature remains neutral with regard to jurisdictional claims in published maps and institutional affiliations. 
Open Access This article is licensed under a Creative Commons Attribution 4.0 International License, which permits use, sharing, adaptation, distribution and reproduction in any medium or format, as long as you give appropriate credit to the original author(s) and the source, provide a link to the Creative Commons license, and indicate if changes were made. The images or other third party material in this article are included in the article's Creative Commons license, unless indicated otherwise in a credit line to the material. If material is not included in the article's Creative Commons license and your intended use is not permitted by statutory regulation or exceeds the permitted use, you will need to obtain permission directly from the copyright holder. To view a copy of this license, visit http://creativecommons. org/licenses/by/4.0/.

\section{References}

1. Hair AB, Peluso AM, Hawthorne KM, Perez J, Smith DP, Khan JY, et al. Beyond necrotizing enterocolitis prevention: improving outcomes with an exclusive human milk-based diet. Breastfeed Med. 2016;11:70-4.

2. Products N, Prolacta F. Nutrition Information 100\%. 2019. https://www.prolacta.com/Data/Sites/14/media/box.com/mkt0627-rev-0_prolacta-product-nutrition-brochure-us_v18.pdf Accessed 5 June 2020.

3. Fenton TR, Nasser R, Eliasziw M, Kim JH, Bilan D, Sauve R. Validating the weight gain of preterm infants between the reference growth curve of the fetus and the term infant. BMC Pediatr. 2013;13:92.

4. Dusick AM, Poindexter BB, Ehrenkranz RA, Lemons JA. Growth failure in the preterm infant: can we catch up? Semin Perinatol. 2003;27:302-10.

5. Lemons JA, Bauer CR, Oh W, Korones SB, Papile LA, Stoll BJ, et al. Very low birth weight outcomes of the National Institute of Child health and human development neonatal research network, January 1995 through December 1996. NICHD Neonatal Research Network. Pediatrics. 2001;107:E1.

6. Clark RH, Thomas P, Peabody J. Extrauterine growth restriction remains a serious problem in prematurely born neonates. Pediatrics. 2003;111:986-90.

7. Abrams SA, Landers S, Noble LM, Poindexter BB. Donor human milk for the high- risk infant: preparation, safety, and usage options in the United States. Pediatrics. 2017;139:e20163440.

8. Sullivan S, Schanler RJ, Kim JH, Patel AL, Trawöger R, KiechlKohlendorfer U, et al. An exclusively human milk-based diet is associated with a lower rate of necrotizing enterocolitis than a diet of human milk and bovine milk-based products. J Pediatr. 2010;156:562-7.e1.

9. Cristofalo EA, Schanler RJ, Blanco CL, Sullivan S, Trawoeger R, Kiechl-Kohlendorfer $\mathrm{U}$, et al. Randomized trial of exclusive human milk versus preterm formula diets in extremely premature infants. J Pediatr. 2013;163:1592-5.e1. https://doi.org/10.1016/j. jpeds.2013.07.011.

10. Eidelman AI, Schanler RJ. Breastfeeding and the use of human milk. Pediatrics. 2012;129:e827-41.

11. Abrams SA, Schanler RJ, Lee ML, Rechtman DJ. Greater mortality and morbidity in extremely preterm infants fed a diet containing cow milk protein products. Breastfeed Med. 2014;9:281-5.
12. Lucas A, Fewtrell MS, Morley R, Lucas PJ, Baker BA, Lister G, et al. Randomized outcome trial of human milk fortification and developmental outcome in preterm infants. Am $\mathrm{J}$ Clin Nutr. 1996;64:142-51.

13. Belfort MB, Ehrenkranz RA. Neurodevelopmental outcomes and nutritional strategies in very low birth weight infants. Semin Fetal Neonatal Med. 2017;22:42-8. https://doi.org/10.1016/j.siny.2016. 09.001 .

14. Radmacher PG, Adamkin DH. Fortification of human milk for preterm infants. Semin Fetal Neonatal Med. 2017;22:30-5.

15. Hair AB, Hawthorne KM, Chetta KE, Abrams SA. Human milk feeding supports adequate growth in infants $\leq 1250$ grams birth weight. BMC Res Notes. 2013;6:459.

16. Patel AL, Engstrom JL, Meier PP, Jegier BJ, Kimura RE. Calculating postnatal growth velocity in very low birth weight (VLBW) premature infants. J Perinatol. 2009;29:618-22.

17. Ebrahim GJ. WHO child growth standards: head circumferencefor-age, arm circumference-for-age, triceps skin fold-for-age and sub scapular skin fold-for-age. J Trop Pediatr. 2007;54:215.

18. Bell MJ, Ternberg JL, Feigin RD, Keating JP, Marshall R, Barton L, et al. Neonatal necrotizing enterocolitis. Therapeutic Decis based Clin staging Ann Surg. 1978;187:1-7.

19. Ehrenkranz RA, Walsh MC, Vohr BR, Jobe AH, Wright LL, Fanaroff AA, et al. Validation of the National Institutes of Health Consensus definition of bronchopulmonary dysplasia. Pediatrics. 2005;116:1353-60.

20. Papile LA, Burstein J, Burstein R, Koffler H. Incidence and evolution of subependymal and intraventricular hemorrhage: a study of infants with birth weights less than 1,500 gm. J Pediatr. 1978;92:529-34.

21. Hair AB, Blanco CL, Moreira AG, Hawthorne KM, Lee ML, Rechtman DJ, et al. Randomized trial of human milk cream as a supplement to standard fortification of an exclusive human milk-based diet in infants $750-1250 \mathrm{~g}$ birth weight. J Pediatr. 2014;165:915-20. https://doi.org/10.1016/j.jpeds.2014. 07.005 .

22. Hair AB, Rechtman DJ, Lee ML, Niklas V. Beyond necrotizing enterocolitis: other clinical advantages of an exclusive human milk diet. Breastfeed Med. 2018;13:408-11.

23. Bushati C, Chan B, Owen AH, Woodbury A, Yang M, Fung C, et al. Challenges in implementing exclusive human milk diet to extremely low-birth-weight infants in a Level III neonatal intensive care unit. Nutr Clin Pract. 2021;1-9.

24. Eibensteiner F, Auer-Hackenberg L, Jilma B, Thanhaeuser M, Wald M, Haiden N. Growth, feeding tolerance and metabolism in extreme preterm infants under an exclusive human milk diet. Nutrients. 2019;11:1443.

25. Visuthranukul C, Abrams SA, Hawthorne KM, Hagan JL, Hair AB. Premature small for gestational age infants fed an exclusive human milk-based diet achieve catch-up growth without metabolic consequences at 2 years of age. Arch Dis Child Fetal Neonatal Ed. 2019;104:F242-7.

26. Ehrenkranz RA, Dusick AM, Vohr BR, Wright LL, Wrage LA, Poole WK. Growth in the neonatal intensive care unit influences neurodevelopmental and growth outcomes of extremely low birth weight infants. Pediatrics. 2006;117:1253-61.

27. Lechner BE, Vohr BR. Neurodevelopmental outcomes of preterm infants fed human milk: a systematic review. Clin Perinatol. 2017;44:69-83. https://doi.org/10.1016/j.clp.2016.11.004. 